\title{
Life Story Development in Childhood: The Development of Life Story Abilities and the Acquisition of Cultural Life Scripts From Late Middle Childhood to Adolescence
}

\author{
Annette Bohn and Dorthe Berntsen \\ Aarhus University
}

\begin{abstract}
The authors investigated the relationship between the acquisition of cultural life scripts and the degree of coherence in children's and adolescents' life stories. Three groups of Danish school children aged 9 to 15 years participated. In 3 sessions, they wrote down a recently experienced single autobiographical event, their life story, and their cultural life script. Single-event and life stories were scored for coherence; life scripts were scored for normativity compared to an adult norm. Single-event stories and life stories were longer and more coherent in the older participants. Younger participants wrote significantly more coherent single-event stories than life stories. When controlling for age, single-event story coherence and global life story coherence did not correlate significantly, suggesting different developmental pathways. Life script normativity increased steadily across childhood and adolescence. Further, a significant relationship between the normativity of life scripts and the coherence of life stories, but not the coherence of single-event stories, was found.
\end{abstract}

Keywords: life story development, acquisition of cultural life scripts, life story coherence

Consider the following narrative provided by a 9-year-old boy in response to the request to tell his life story: "I got lost from my mum and dad on the beach. And they had to ask other people to help search for me. And then they finally found me down on the beach, and then we had to go home." Instead of a life story, he provides an account of a single emotional event.

There is ample empirical evidence that children are able to remember autobiographical events from quite an early age and that their ability to narrate these memories improves over the preschool and early school years (e.g., Fivush, Haden, \& Adam, 1995; Hamond \& Fivush, 1991; van Abbema \& Bauer, 2005; see Nelson \& Fivush, 2004; Peterson, 2002; Reese, 2002, for overviews). However, not much is known about children's ability to combine narratives of individual autobiographical memories into a coherent life story. In fact, there is no empirical evidence for the existence

Annette Bohn and Dorthe Berntsen, Department of Psychology, Aarhus University, Århus, Denmark.

The study reported in this article was conducted in partial fulfillment of the requirements for the doctoral degree in the Department of Psychology at the University of Aarhus for Annette Bohn. It was supported by a doctoral stipend from the Faculty of Social Sciences at the University of Aarhus.

We thank the teachers and especially the students at Rønde School for their participation in this study and for sharing their life stories with us. We are thankful to Karen Bødker for establishing contact with Rønde School. We thank Kim Berg Johannessen and Meike Bohn for scoring the data, Anne Stærk Jacobsen for transcribing the cultural life scripts, Bo Sommerlund for statistical assistance, and the Danish Research Council for Culture and Communication for funding parts of the project.

Correspondence concerning this article should be addressed to Annette Bohn, Department of Psychology, University of Aarhus, Jens Chr. Skous Vej 4, 8000 Århus C., Denmark. E-mail: anetboh@psy.au.dk of life stories in children, and it has been proposed that the ability to construct a coherent life story is not developed before adolescence or young adulthood (Habermas \& Bluck, 2000; McAdams, 2001; Singer, 2004). However, little empirical evidence has been presented to support this claim.

Research on life narratives in adults (e.g., Luborsky, 1993; McAdams, 1996, 2001) suggests that these are formed by the individual in close interaction with his or her social and cultural environment (Nelson, 2003; McAdams, 2001; Singer \& Bluck, 2001). McAdams (2001) defined life narratives as "psychosocial constructions coauthored by the person himself or herself and the cultural context within which that person's life is embedded and given meaning" (p. 101). Thus, according to this view, life narratives are made up of autobiographical memories that are selected by the individual to form a coherent personal narrative in the framework of a given cultural context. The present work examines the age at which children show the ability to produce a coherent life story and the extent to which the development of this ability is mediated by the acquisition of shared cultural knowledge of prototypical life events and their timing. The following is a review of the literature that pertains most directly to the present work.

\section{Coherence in Life Stories}

Habermas and Bluck (2000) gave a detailed theoretical account of the development of the necessary cognitive abilities and motivation to create a coherent life story. According to their theoretical claims, such ability is most likely to develop through childhood and well into adolescence. They argue that a coherent life narrative needs to be based on autobiographical reasoning, that is, the ability to create coherence between the remembered autobiographical events and to mold them into a meaningful story. Without autobiographical reasoning, a life narrative would only consist of lists 
of isolated autobiographical memories. Further, the ability of autobiographical reasoning leads to the emergence of global coherence in the life narratives of adolescents, which consists of four different types of coherence: temporal, thematic, causal, and cultural.

Temporal coherence refers to the ability to order events chronologically. In a coherent life narrative, the temporal sequence of events needs to be respected; for example, it would be considered incoherent to mention a job promotion before mentioning getting a job. According to the literature reviewed by Habermas and Bluck (2000), basic temporal coherence is acquired across the preschool years (e.g., Friedman, 1992; McCormack \& Hoerl, 1999; Nelson, 1988). However, the ability to order autobiographical events by the seasons of the year first develops from about 9 years of age. By age 13, a majority of children have acquired this ability (Friedman \& Lyon, 2005). Thematic coherence refers to the ability to create overarching themes in a narrative and to establish thematic similarities between various events. Thus, to tell a thematically coherent life narrative implies the ability to see one's life in the framework of life themes or to see different autobiographical events as typical for one's life. Habermas and Bluck proposed that thematic coherence emerges in midadolescence. Causal coherence is the type of coherence that develops the latest, namely in middle or late adolescence (Habermas \& Paha, 2001). A causally coherent life narrative is told in terms of motives and causes. Through causal coherence, reason and meaning are added to the life narrative, and it facilitates an explanation of how one has become the person one is. Causal coherence is closely interwoven with the establishing of an identity during adolescence (Erikson, 1968).

The fourth type of coherence, cultural coherence, refers to "shared normative expectations concerning appropriate and probable life sequences" (Habermas \& Bluck, 2000, p. 750). Habermas and Bluck (2000) theorized that cultural coherence (which they also called "the cultural concept of biography") is used-together with temporal coherence- " consisting of an ordered sequence of culturally defined, major life events" (p. 750). This definition of a cultural concept of biography is built on well-established findings from sociological and anthropological research that there exist cultural norms and values in every culture that prescribe the events that are expected to happen at certain points in time in a prototypical life in that culture (for overviews, see Berntsen \& Rubin, 2002, 2004; Habermas \& Bluck, 2000; Neugarten, Moore, \& Lowe, 1965). According to Habermas and Bluck, cultural coherence may vary across cultures. Further, they assume cultural coherence to be specific to life narratives, whereas the other three types of coherence (temporal, causal, and thematic) are assumed to be general linguistic types of coherence to be found in all narratives. In the present study, cultural coherence is measured outside of and separately from life stories in the form of the cultural life script. Hereby, cultural coherence is measured separately from the other three types of coherence, which can only be measured within narratives. Obtaining an independent measure of cultural coherence is especially important because it allows us to examine Habermas and Bluck's assumption that cultural coherence is the only type of coherence that is specific to the life story. The cultural life script is described and discussed in more detail, as it is one of the foci of the current study.

\section{Cultural Life Scripts}

The concept of the cultural life script by Berntsen and Rubin (2004) encompasses the notion of a cultural concept of biography (Habermas \& Bluck, 2000) and has been operationalized successfully and reliably across various studies and populations (Berntsen \& Rubin, 2004; Erdoğan, Baran, Avlar, Cağlar Taş, \& Tekcan, 2008; Rubin \& Berntsen, 2003; Rubin, Berntsen, \& Hutson, 2008). The cognitive structure of the cultural life script combines the concept of story scripts as described by Schank and Abelson (1977) with results from research on life course development of cultural norms for the timing of salient life events (for an overview, see Berntsen \& Rubin, 2002, 2004; Neugarten et al., 1965). Berntsen and Rubin (2004) clearly differentiated between life narrative and cultural life script. Life narratives are made up of autobiographical memories as they are remembered and reconstructed by an individual (under the influence of the "guidelines" provided by cultural life scripts). Cultural life scripts are semantic knowledge. They refer to the culturally shared representations of an idealized life. Cultural life scripts teach an individual how a life in his or her culture should ideally be lived. A cultural life script can be described as a series of important transitional events that should happen in a set temporal order (e.g., "finish school" before "getting married"). Each event (e.g., marriage) is allocated a specific time slot in the life course, referring to the age at which the event is normatively expected to take place (e.g., marriage around age 27). The cultural life script is learned detached from personal experience, that is, one need not have experienced an event personally to nominate it as an important transitional event with the appropriate time slot (e.g., young adults have no difficulty in nominating "having children" or "retirement" as cultural life script events with specific time slots, despite the fact that they have not experienced these events). Individual lives as actually lived may deviate from the normative timing for various personal reasons. Such deviations would often need to be accounted for in the personal life narrative (e.g., "We married late because my husband served in the army during the war"). In a similar vein, Schank and Abelson differentiated between the underlying script of a story (e.g., the subway script), which is automatically used to reconstruct a story, and all unexpected events within a story (akin to life events deviating from the cultural life script), which are "placed on a 'weird list' to be specially remembered" (Schank \& Abelson, 1977, p. 429). As the emphasis in life scripts is on the idealized life, life scripts are expected to be dominated by positive events.

Personal experiences that correspond to events in the cultural life script (e.g., graduation) are likely to be appreciated as important when they take place because they come with a meaning that is culturally agreed upon, notably as transitional events. The focus in the cultural life script on social transitional events can facilitate rehearsal in various ways: The events are likely to be rehearsed prospectively and retrospectively; that is, people tend to talk and think about upcoming transitional events in their lives, as well as events that have happened in their past. This rehearsal can be both private and social and is often supported by the existence of external records of the event, such as photos and videos.

\section{Cultural Life Scripts and Development}

Why would the acquisition of a cultural life script be important to the ability to narrate one's personal life? Cultural life scripts 
provide an outline or "search machine" for the recall of autobiographical events that are essential for the construction of a life story. In other words, a cultural life script is a generic structure on the basis of which concrete personal life stories can be generated in the same way as specific stories about eating at a restaurant can be generated from a generic restaurant script (Schank \& Abelson, 1977). However, whereas Schank and Abelson's (1977) story scripts were assumed to be acquired through personal experience, cultural life scripts are learned through cultural transmission, according to Berntsen and Rubin (2004). Through this transmission, the child gradually learns how a life ideally is lived in his or her culture and builds up a script for the culturally expected segmentation of the life course into life time periods demarcated by normative transitional events. The cultural life script can thus be seen as a prototypical (or stereotypical) life story that children need to learn to have a model for their own personal life stories, although the latter is likely to deviate some from the prototype. In a broader sense, through the cultural life script, children acquire knowledge about what they can expect of life and what is expected of them as a member of the culture in which they grow up. They learn to view their personal past and future through the eyes of their culture. The idea of the cultural life script as a precondition to life story abilities therefore agrees well with the notion of cultural learning as a unique and important vehicle for the social and cognitive development of the human child (e.g., Tomasello, 1997; Tomasello, Kruger, \& Ratner, 1993) as well as the related view that the development of autobiographical memory to a great extent relies on social and cultural mechanisms (Nelson \& Fivush, 2004).

\section{Evidence for Cultural Life Scripts}

Research has found evidence for the existence of stable cultural life scripts dominated by positive events in populations of young Danish and American adults (Berntsen \& Rubin, 2004; Rubin \& Berntsen, 2003; Rubin et al., 2008) as well as in young Turkish adults (Erdoğan et al., 2008). Berntsen and Rubin (2004) asked 103 Danish psychology majors to generate the seven most important events they thought would occur in a prototypical newborn infant's life. Participants were also asked to estimate the age at which the events would occur in the infant's life. Berntsen and Rubin (2004) found strong agreement among participants as to the kind of events that would happen to the infant across the life span. Close to $95 \%$ of all events mentioned could be fit into 1 of 35 categories. Also, the majority of events were judged to be positive and to occur in the time span from 15-30 years. In a study involving Danish and American participants, Rubin et al. (2008) replicated the findings on the cultural life scripts in the Danish sample and also found the existence of a cultural life script in the American sample. The American life script resembled the Danish one, but showed, as should be expected, some cultural variation (e.g., getting a driver's license was part of the American life script but not of the Danish one; for a more detailed discussion of life script differences, see Rubin et al., 2008). The participants were also asked to write down the seven most important events from their own lives that they would include in their personal life stories. In both groups of participants, a substantial overlap was found between the events mentioned for the personal life stories and events mentioned in the cultural life script.
In a recent study, Collins, Pillemer, Ivcevic, and Gooze (2007) found evidence that the cultural life script indeed is used as a kind of search machine for autobiographical memories. Collins et al. asked 92 American participants (average age $=21$ years) to describe two positive and two negative memories from the time when they were between 8 and 18 years old. They found negative memories to be flatly distributed across the years, whereas positive memories were clustered around life script events, such as finishing high school and beginning college. About half of all positive memory events happened around ages 17-18. When Collins et al. repeated the study for an age range that (according to their estimate) does not include prominent cultural life script events in an American population (ages 10-15), a flat distribution for both positive and negative memories was found. ${ }^{1}$ A recent study by Erdoğan et al. (2008) investigated the cultural life script in a Turkish sample of 200 university students, who were, on average, 20 years old. The study looked specifically at possible gender differences in the cultural life script. Erdoğan et al. asked their participants to generate two different life scripts: one for a person of their own gender and one for a person of the opposite gender. The results showed no differences between the life scripts generated by men and women, and also, there were no gender differences between the life scripts generated by women for males and life scripts generated by men for females.

\section{Developmental Studies of Cultural Life Scripts and Coherence in Life Stories}

Several studies have found that children's narratives of single autobiographical events become more complex and structured across the preschool and early school years (e.g., Fivush et al., 1995; Haden, Haine, \& Fivush, 1997; Hudson \& Fivush, 1991; Peterson \& McCabe, 1983; Pillemer, Picariello, \& Pruett, 1994; van Abbema \& Bauer, 2005). However, very few studies have examined Habermas and Bluck's (2000) claims that a coherent life story first begins to emerge in adolescence and that cultural life scripts are the scaffolding necessary for the construction of coherent life stories. Instead, the validity of these claims has often been taken for granted (McAdams, 2001, 2006; McLean, 2005). In the following, we first describe one study looking at the development of some aspects of the cultural concept of biography and then describe findings from studies related to the development of coherence in life stories.

Habermas (2007) studied the development of biographical salience and age estimation norms for life events in a German sample of four groups of children and adolescents (age range $=8-21$ years). Results showed that performance on the age estimation and salience tasks were positively correlated, and significant effects of age were found, with 8.5-year-olds performing most poorly on both tasks and 12.5-year-olds performing significantly lower on the age estimation tasks than the older groups. Thus, this study provides empirical evidence that children acquire an idea of the biographical salience of events earlier than the age norms of age-graded events. However, this study does not provide much information on the acquisition of cultural life scripts as operation-

\footnotetext{
${ }^{1}$ The Danish life script contains a prominent event in this range, namely confirmation (see the results from the present study described later).
} 
alized by Berntsen and Rubin (2004), because the children were not asked to generate life script events and because the participants were asked to decide whether a specific event should be included in the life story of a friend, instructions that are distinctly different from the instructions used to elicit life scripts.

Habermas and de Silveira (in press) conducted an interview study on linguistic coherence in guided life narratives. Participants were 102 young Germans who were divided into four age groups $(8.5,12.5,16.5$, and 20.5 years old). Prior to telling life narratives, participants were asked whether they had experienced any of 14 primarily negative life events. Further, they were asked to write down the seven most important memories from their lives and to order these memories chronologically. Afterward, participants were explicitly asked to include the seven events in their life narratives. Participants were interviewed twice over a 2-week delay to test for training effects on narrative ability. Life narratives were coded for seven different temporal, causal, and thematic linguistic indicators of coherence that had been developed in an exploratory study (Habermas \& Paha, 2001). Further, fluid and crystallized intelligence and frequency of biographical practices (such as keeping a diary) were measured. Finally, life narratives were scored by the listeners on three scales ranging from 1-7 on overall temporal, causal, and thematic coherence. Habermas and de Silveira found that the percentage of temporal, causal, and thematic indicators of coherence increased with age. Also, listeners' ratings of narrative coherence increased across the age groups. Training, intelligence, and frequency of biographical practice played no role for the linguistic coherence of the life narratives.

In a 3-year longitudinal study involving only young adultscohorts of college freshmen and seniors (age range over 3 years $=$ 18-25 years) — McAdams et al. (2006) asked college students in a guided autobiography procedure (de Vries, Birren, \& Deutchman, 1990) to write down two memories each of high points, low points, and turning points in their lives, as well as two early memories and two other memories from times in their lives not mentioned in the other eight memories. Further, the students were explicitly instructed to think and write about what they were thinking and feeling during the event, why this event was important for their life stories, and what this event might "say about who you are, who you were, who you might be, or how you have developed over time" (McAdams et al., 2006, p. 1379). The students wrote the 10 key scenes from their life stories at Time 1, 3 months later, and 3 years later. The narrative complexity of the autobiographical memory accounts (measured dichotomously as either present or absent) increased significantly within participants over the years. From these studies, there seems to be some empirical evidence that narrative coherence increases throughout adolescence and into early adulthood. However, one important limitation of both studies is that in none of the studies is it possible to discern whether students' development of narrative coherence is specific to life stories or whether the participants simply improved their narrative abilities in general, because cultural coherence, which is presumed to be specific to life narratives (cf. Habermas \& Bluck, 2000), was not measured. This is a highly relevant problem, among other things, because participants in the first study (Habermas \& de Silveira, in press) were academically advanced junior high and high school students, whereas the participants in McAdams et al.'s study were college students. Thus, both groups of participants were trained over years to refine exactly those skills that were measured in these two studies. In short, neither of the two studies examined the development of life story coherence against a baseline measure of narrative coherence. Such a baseline is needed to disentangle the development of life story abilities from the acquisition of general narrative skills. In the current study, we include such a baseline measure by having the participants not only write their life stories but also write about an autobiographical event from their recent past. Further, we collect entire, unguided life stories to measure the degree of global coherence in life story narratives (i.e., the combination of all four types of coherence; $\mathrm{cf}$. Habermas \& Bluck, 2000), and we collect cultural life scripts to measure cultural coherence independently of life story coherence. Hereby, it is possible to investigate whether the acquisition of cultural life scripts is instrumental for being able to construct one's life story.

\section{The Present Study}

The present study addresses three major questions: At what age are children or adolescents able to write a coherent life story? At what age are cultural life scripts acquired? Is there a relationship between the acquisition of a cultural life script and the ability to compose a coherent life story? On the basis of earlier research on adolescents' and young adults' coherence in autobiographical memory narratives (Habermas \& de Silveira, in press; Habermas \& Paha, 2001; McAdams et al., 2006), we expect an increase of coherence across age groups for narratives of single autobiographical events as well as for life stories. On the basis of an earlier study (Habermas, 2007), we expect life scripts to become more adult-like across age groups. On the basis of theory (Habermas \& Bluck, 2000) and evidence that adults use cultural life scripts as scaffolds to construct a life story (Berntsen \& Rubin, 2004; Collins et al., 2007; Rubin et al., 2008), we expect that adolescents with a more normative or adult-like cultural life script will write more coherent life stories than adolescents with a more idiosyncratic, less normative life script. We do not expect a relationship between the normativity of the life script and coherence in stories about a single autobiographical event because the knowledge of a cultural life script has been specifically linked with the construction of life stories (Berntsen \& Rubin, 2004; Habermas \& Bluck, 2000).

From a linguistic point of view, both written and oral elicitations of life stories are equally feasible because analyses of discourse patterns of teenagers and young adults have not found any differences in the linguistic coherence of written versus oral narratives (Smith, Heuerman, Wilson, \& Proctor, 2003; Wilson, Smith, \& Proctor, 2001). Eaton (2005) compared data from different studies involving audiotaped, interviewed, handwritten, and typed narratives by young adults about positive and negative autobiographical events. She found no difference in the length of handwritten and oral narratives. However, in written narratives, compared with narratives elicited by interviews, participants used more cognitive processing words when reporting negative events than when reporting positive events; the same amount of cognitive processing words were used, regardless of valence, when participants were interviewed about their experiences. Also, in the interview condition, fewer affect terms were used than in the written narratives. Therefore, according to Eaton, "written and typed narratives appear to be more complex and cohesive compared with spoken accounts" (Eaton, 2005). This might indicate that written autobio- 
graphical accounts facilitate a more thorough, private and truthful reporting of emotional autobiographical events. For the eliciting of life stories in older children and teenagers, the method of written accounts might be preferable because writing one's life story anonymously versus being interviewed by an adult stranger leads to the possibility of a much more open account, unrestrained by social considerations. As indicated by the linguistic literature, there are no differences concerning narrative coherence of oral versus written accounts. However, from a psychological point of view, written life stories might be more open, truthful, and even self-critical. Thus, we use written accounts in the present study.

\section{Method}

\section{Participants}

A total of 140 Danish middle-class children with a culturally homogenous background participated in the study. Participants were recruited from a typical Danish comprehensive school (from kindergarten through ninth grade) in a midsized town. Participants who did not attend the data collection session for cultural life scripts (see the Procedure section below) or who did not follow instructions were excluded from the study, leaving a total of 120 participants.

Data were collected from children in the third, fifth, sixth, and eighth grades. Children in the fifth and sixth grades formed one group for two reasons: There were very few students in the fifth and sixth grades, and the age ranges overlapped in these two groups (fifth-grade age range $=10$ years, 8 months to 12 years, 11 months; sixth-grade age range $=12$ years, 0 months to 12 years, 10 months). Thus, the sample was divided into three groups: 42 third graders ( 26 boys; mean age $=9.51$ years, $S D=0.28$ ), 43 fifth-sixth graders ( 23 boys; mean age $=11.87$ years, $S D=0.59$ ), and 35 eighth graders (16 boys; mean age $=14.46$ years, $S D=$ $0.30)$.

To measure the acquisition of cultural life scripts, we established a norm by collecting cultural life scripts from an adult group of Danish psychology majors $(N=111 ; 34$ men; mean age $=$ 28.33 years, $S D=6.72$ ). The psychology students were recruited during a lecture on organizational psychology and during lunch time hours at the campus cafeteria.

\section{Procedure}

Parents had been informed in advance through a letter by the school principal about the study, asking them to call or send a note if they did not want their children to participate. None of the parents denied their child's participation.

Data collection took place during regular Danish classes during school hours in each individual class grade. In three sessions of 45 min each, the following data were collected from the children over a 3-week period: (a) a written report of a recent autobiographical event, (b) a written life story, and (c) a cultural life script. The cultural life script task was presented last to prevent this task from influencing how children solved the other two tasks. The interval between the three tasks ranged from 7 to 16 days between the first and second visit and 5 to 14 days between the second and third visit. The total time passing between the first and last visit ranged from 18 to 21 days.
For the written report of a recent autobiographical event, the children were asked to write about an event that had happened during their fall vacation, which had ended about 3 weeks before data collection took place. This ensured that participants wrote about similar recent, relatively unemotional, events happening at about the same time. The single-event stories were used as a baseline measurement for the children's ability to coherently narrate an autobiographical event.

For the written life story, participants received the following instructions:

Think about what has happened in your life since you were born and up to now. You can for example write about the most important things in your life, or what changes have happened in your life since you were born and up to now. You can also tell about other things you have experienced. Imagine that you are writing the story of your life to someone who would like to know something about you and the things that have happened in your life.

For the cultural life script, participants were asked to imagine a newborn of their own sex and to write down the 10 most important events that they thought would happen in the newborn's life across the life span. (We used 10 events instead of the seven used in earlier studies to increase variability on the level of performance.) They were further asked to estimate how old the newborn would be when these events occurred. Participants were told that there were no right or wrong answers for this task but that we were interested in their opinions.

For all three tasks, the children received a booklet with a cover page asking for their name and the languages spoken at home. The participants' ages were obtained from school records. It was explained to the children that their names were used only to match their three sets of data and that their data would be anonymized. They were also told that participation was voluntary and that they could stop their participation at any time during data collection. The experimenter read the instructions printed in the booklets to the class and answered any questions. The participants were told that they should not worry about spelling mistakes or handwriting when writing their stories.

\section{Scoring}

Cultural life scripts. First, the life scripts from the adult group were scored to set an adult norm. The instructions for the adult participants were the same as the instructions described above for the children concerning the cultural life script. The life script events were classified according to the 35 categories established by Berntsen and Rubin (2004). Events that did not fit these categories and were mentioned four times or more were identified as new categories. All events mentioned three times or less were scored as "other." This procedure resulted in a total of 42 event categories. Thirty percent (36 of 111) of all adult life scripts were scored by two raters. They agreed on $96 \%$ of all categories. Disagreements were resolved by discussion. The remaining adult life scripts were scored by one rater. The children's life scripts were scored according to the categories generated from the adult life scripts. If children mentioned an "other" event that was also mentioned by an adult, this event was scored as "other adult." All other events that were not mentioned by at least one adult were scored as "other child." This method was used even when more 
children had mentioned a specific life script event, as we were interested in the development of children's life scripts compared with an adult norm (the bottom section of Table 3 shows events mentioned by 3 or more children that were not included in the adult life script). Two raters scored $54 \%$ of all life scripts following these guidelines. They agreed in $94 \%$ of all events. Differences were resolved by discussion. The remaining $46 \%$ of life scripts were scored by one rater.

Two scores were developed to measure life script normativity: a typicality score and an idiosyncrasy score. The typicality score was based on how often a life script event had been mentioned by the adult group. For example, the life script event "fall in love" received the typicality score 59 , as it was mentioned by 59 of the 111 adult participants. The typicality scores ranged from 100 ("having children") to 4 ("become a grown up"). "Other adult" events received a score of 2 and "other child" events a score of 1 . Thus, the higher a participant scored on the typicality score, the more normative his or her life script is considered. To simplify, "other adult" and "other child" are labeled "idiosyncratic events" in the following. The idiosyncrasy score measured individual variation across life scripts by counting the number of idiosyncratic events mentioned in the life script. Idiosyncratic events are all those events that were mentioned by less than 4 adult participants and thus were scored as "other adult" or as "other child."

Coherence. Children's single-event stories were scored on global coherence using a scale from 0 to 3 and adapted from Peterson and McCabe's (1983) scale for the scoring of single autobiographical event stories (see also Fivush, Hazzard, McDermott Sales, Sarfati, \& Brown, 2003), with $0=$ disoriented pattern (the narrative is too confused or disoriented for the listener to understand), 1 = chronological pattern (the narrative is a simple description of successive events ["and then, and then"]), $2=$ chronological, interesting pattern (the narrative describes successive events in detail and interestingly; e.g., use of adjectives, adverbs, and subordinate clauses), and $3=$ classic pattern (the narrative builds to a highpoint, evaluatively dwells on it, then resolves it). ${ }^{2}$

Scorings for 0,1 , and 3 were taken directly from Peterson and McCabe's (1983) scale, whereas scoring for 2 was adapted to our data material because our material did not contain any stories with an "ending-at-the-highpoint pattern," as described in Peterson and McCabe's scoring for 2 (Peterson \& McCabe, 1983, p.37). Instead, our data material contained chronological stories that were clearly more sophisticated than the chronological stories with a scoring of 1. Following procedures used by Fivush et al. (2003), two raters independently coded $25 \%$ of the single-event stories and achieved $87 \%$ agreement. Disagreements were solved by discussions. One rater scored the remaining stories.

As life stories are more complex and involve more than one autobiographical event memory, life story coherence was measured by the following parameters: (a) a scale rating the way in which the life story begins, (b) a scale rating the way the life story ends, (c) a scale measuring global life story coherence, and (d) number of life script events mentioned in the life story. The scales measuring beginning and ending of the life stories were adapted from Habermas (2006). For the beginnings of life stories, the scores were $0=$ begins some time after birth, $1=$ begins with birth, 2 = begins with birth plus own name or place of birth, and $3=$ begins with birth plus name-place and date of birth. For the ending of life stories, the scores were $0=$ ends in the past, $1=$ ends in the present, 2 = ends with short resume and/or outlook into the future, and $3=$ ends with elaborate resume and/or outlook into the future. For the global life story coherence, we took Peterson and McCabe's (1983) scale for single-story coherence as a starting point to develop a scale that was parallel but at the same time adjusted to the circumstance that life stories by definition consist of more than a single autobiographical event story. The scores were $0=$ life story consists of a single episode; $1=$ several episodes, loosely ordered chronologically; 2 = chronological order, episodes are tied together; and 3 = chronological, evaluative narrative. In addition, the length of single-event stories and life stories were measured by word count.

The number of life script events in life stories was scored by counting all those events mentioned by the children in their life stories that were part of the adult life script. Also, to be able to compare the content of individual life stories with the matching individual life script, we listed all events mentioned in the life stories. This included events that were neither part of the adult life script norm nor mentioned by any of the children as life script events. Examples of such events are "birth of a cousin" or "sibling gets in trouble with the law." For life story variables, two raters scored $25 \%$ of all life stories. Their agreement rates were $96 \%$ for beginning of life stories, $83 \%$ for ending of life stories, $90 \%$ for global life story coherence, and $89 \%$ for frequency of life script events in life stories. Cases with disagreement were resolved by discussion. The remaining life stories were scored by one scorer.

\section{Results}

First, we present the results concerning the children's life stories and single-event stories. Then findings for cultural life scripts are presented. Finally, we present analyses concerning the relationship between cultural life script acquisition and the coherence of singleevent and life stories.

\section{Life Stories and Stories of a Single Event}

Life stories. In a series of analyses of variance (ANOVAs), we compared the means of the life story length, global life story coherence, life story beginnings, and life story endings and the frequency of life script events in the life story across the three child groups (see Table 1). We found clear and highly significant developmental differences for all variables, with life stories becoming longer and more coherent with age: length, $F(2,109)=$ 109.26, partial $\eta^{2}=.667, p<.0001$, confidence interval $(\mathrm{CI})=$ 184.99-244.19; global coherence, $F(2,109)=46.96$, partial $\eta^{2}=$ $.463, p<.0001, \mathrm{CI}=1.28-1.61$; beginning, $F(2,109)=19.03$, partial $\eta^{2}=.259, p<.0001, \mathrm{CI}=1.12-1.53$; ending, $F(2,109)=$ 31.04 , partial $\eta^{2}=.363, p<.0001, \mathrm{CI}=0.74-1.06$; and frequency of life script events, $F(2,109)=42.61$, partial $\eta^{2}=.439$, $p<.0001, \mathrm{CI}=3.27-4.07$. Post hoc Tamhane's tests showed that for all variables except for beginning of life stories, there were significant differences between all three groups of children ( $p \mathrm{~s}$ between .02 and .0001). For the beginning of life stories, the

\footnotetext{
2 Peterson and McCabe (1983) used the term "event" in their scoring system as a description for actions within a single autobiographical event story.
} 
Table 1

Means (and Standard Deviations) for Length of Single-Event Stories and Life Stories, Coherence Variables, and Cultural Life Script Variables in the Three Child Groups

\begin{tabular}{lcc}
\hline \multicolumn{1}{c}{ Variable } & Third grade & Fifth-sixth grade \\
\hline Single-event story length & $92.00(41.20)$ & $136.97(74.96)$ \\
Life story length & $83.43(36.01)$ & $194.38(97.25)$ \\
Beginning life story (0-3) & $0.58(0.78)$ & $1.70(1.16)$ \\
Ending life story (0-3) & $0.28(0.45)$ & $1.03(0.80)$ \\
Frequency of life script events in life stories & $1.95(1.50)$ & $3.95(1.71)$ \\
Single-event story coherence $(0-3)$ & $1.20(0.61)$ & $1.78(0.87)$ \\
Global life story coherence $(0-3)$ & $0.78(0.53)$ & $1.53(0.76)$ \\
\hline
\end{tabular}

Note. Values for both vacation story and life story lengths are word counts.

results showed that both older child groups were more likely to begin their life stories with their birth, rather than at some point after birth, contrary to the third graders (see Tables 1 and 2). Measures of length and coherence of life stories correlated with age, ranging from $r(109)=.458$ for beginnings of life stories to $r(109)=.808$ for length of life stories (all $p \mathrm{~s}<.0001)$.

Table 2 presents the results of a series of chi-square tests for beginning and end of life stories and global life story coherence in the three child groups. The table shows that $60 \%$ of all third graders began their life story at some point after their birth, and almost three fourths of them ended their life story suddenly at some point in the past. Also, $28 \%$ of third graders clearly seemed to lack the concept of a life story, as they reported only a single, isolated episode from their lives, such as the example of the 9 -year-old boy getting lost quoted at the beginning of this article.

By the fifth grade, only 1 child reported a life story consisting of a single episode. Overall, an inspection of Table 2 indicates that none of the children in the third grade were able to provide their life stories, children in the fifth-sixth grade showed an ability to narrate a series of events from their lives in some detail and to some extent organize them chronologically, and a substantial minority of children in the eighth grade were able to provide a detailed life story with an evaluative and chronological structure as well as appropriate beginnings and endings.

Single-event stories. Findings related to stories about the children's vacation are also presented in Table 1. As expected, children wrote about similar, relatively unemotional or slightly positive events from their vacations. The majority of stories concerned family vacations or outings and experiences with friends (i.e., going shopping, going to the movies, playing computer games or soccer).

A series of ANOVAs showed age-related differences in the length and coherence of single-event stories, with length and coherence increasing over age: length, $F(2,109)=32.97$, partial $\eta^{2}=.377, p<.0001, \mathrm{CI}=132.70-172.57$; and coherence, $F(2$, 109) $=15.83$, partial $\eta^{2}=.225, p<.0001, \mathrm{CI}=1.45-1.71$. Post hoc Tamhane's tests showed that the two older child groups wrote significantly longer single-event stories than did the third graders. For the coherence of the single-event stories, the tests indicated clear differences between all three groups, in terms of increasing coherence from third to eighth grade (see Table 1). Length and coherence of single-event stories also correlated with age, $r(109)=.580$ and $r(109)=.464$, respectively $(p s<.0001)$

Table 2

Scores of Beginning and Ending of Life Stories and Global Life Story Coherence in the Third, Fifth-Sixth, and Eighth Grades (Percentage of Children in Each Group)

\begin{tabular}{|c|c|c|c|c|}
\hline Variable & Third grade & Fifth-sixth grade & Eighth grade & $\chi^{2}$ \\
\hline Beginning of life story & & & & $42.48^{* * * *}$ \\
\hline Sometime after birth & 60.0 & 25.0 & 15.6 & \\
\hline With birth or age & 22.5 & 10.0 & 3.1 & \\
\hline With birth and name-place & 17.5 & 35.0 & 68.8 & \\
\hline With birth plus date and name-place & 0.0 & 30.0 & 12.5 & \\
\hline Ending of life story & & & & $49.15^{* * * *}$ \\
\hline Suddenly in the past & 72.5 & 25.0 & 9.4 & \\
\hline In present & 27.5 & 52.5 & 34.4 & \\
\hline Short resume-outlook to future & 0.0 & 17.5 & 50.0 & \\
\hline Elaborate resume-outlook to future & 0.0 & 5.0 & 6.3 & \\
\hline Global life story coherence & & & & $60.71^{* * * *}$ \\
\hline Isolated episode & 27.5 & 2.5 & 0.0 & \\
\hline Several episodes & 67.5 & 57.5 & 12.5 & \\
\hline Chronological, detailed & 5.0 & 30.0 & 50.0 & \\
\hline Evaluative, chronological & 0.0 & 10.0 & 37.5 & \\
\hline
\end{tabular}

**** $p<.0001$. 
An inspection of Table 1 shows large differences in the scores of third graders for single-event story coherence and global life story coherence. We conducted paired $t$ tests for all three child groups with the two global coherence variables and found that third graders (but not fifth-sixth and eighth graders) scored significantly lower on global life story coherence than on single-event story coherence, $t(37)=3.42, p<.005$ (CI for the difference $=$ -0.67 to -0.17 ). Also, there was no significant correlation between global life story coherence and single-event story coherence when controlling for age of the participants, $r(103)=.175, p>.05$. The ratio of length of life stories to length of single-event stories seemed to increase across age groups. We explored this possibility by generating a new ratio variable by dividing the length of single-event stories into the length of the life stories. A one-way ANOVA with this ratio as the dependent variable and the three groups as factor found a main effect of group, $F(2,103)=14.41$, partial $\eta^{2}=.219, p<.0001, \mathrm{CI}=1.33-1.64$. Post hoc Tamhane's tests showed that fifth-sixth graders and eighth graders wrote significantly longer life stories than single-event stories compared with third graders (third grade: $M=1.06, S D=0.55$; fifth-sixth grade: $M=1.55, S D=0.55$; eighth grade: $M=1.97$, $S D=0.98)$.

\section{Cultural Life Scripts}

Table 3 shows the adult life script events, their frequencies of recording in percentages, and the estimated ages at occurrence with standard deviations for the adults and the three groups of children (top section). The adult life script presented in Table 3 largely replicates Berntsen and Rubin's (2004) study. The few differences that can be observed most likely reflect that Berntsen and Rubin asked their participants for seven life script events, whereas the participants in the present study were asked to generate 10 life script events. The present life script contains 42 categories, compared with 35 categories (not counting "others") in Berntsen and Rubin's study. The majority of new categories consisted of more differentiated versions of the same overall categories (e.g., "begin in daycare" was differentiated into "begin in nursery" and "begin prekindergarten"). Six categories from Berntsen and Rubin's study did not reach category status in the present study. Only one of these ("go to school") was among the top 20 events in Berntsen and Rubin's study and was replaced by more differentiated categories in the present study ("finish ninth grade," "begin high school," "finish high school," and "continuation school"). The new event categories in this study were exclusively neutral and positive events from the first three decades of life, with the exception of the category "second-last child," which was estimated to occur at age 31 (see Table 3). For the 27 events that directly overlapped with Berntsen and Rubin's life script events, the recording frequencies and age estimates were highly correlated with the ones reported by Berntsen and Rubin ( $r s=.94$ and .99 , respectively, $p s<.0001$ ). In short, the present agreement in life event categories, their frequencies, and estimated ages agree well with findings reported in earlier studies.

The life script events generated by the three groups of children are also presented in Table 3 . The individual child groups were smaller than the adult norm group; therefore, we included events that were mentioned by at least 3 children per group (vs. mentioned by at least 4 persons in the adult group). The top section of
Table 3 shows the recording frequencies in percentages and estimated ages for the 42 life script events that were generated by the adult norm group, whereas the bottom section includes events that did not make it into the adult life script but were nonetheless mentioned by 3 or more children in each child group. As shown in the top section, in all three groups of children some of the adult event categories were not mentioned by at least 3 participants. As shown in the bottom section, the number of nonadult event categories diminishes with increasing age. To examine how well the recording frequencies and estimated ages of children's life script events agreed with the ones of the adult norm group, we correlated the relevant columns in Table 3 (under the assumption that these measures could be treated as independent observations). For the third graders, the $r$ s were .55 and .98 for recording frequencies and ages, respectively (overlapping categories $=26, p \mathrm{~s}<.01$ ). For the fifth-sixth graders, the corresponding $r$ s were .80 and .99 (overlapping categories $=26, p \mathrm{~s}<.0001)$. For the eighth graders, the $r$ s were .78 and .95 (overlapping categories $=27, p s<.0001$ ). Thus, the estimated ages are extremely highly correlated with the ones in the adult norm group; indeed, the correlations are as high as the one obtained when we correlated age norms in Berntsen and Rubin's (2004) study with the adult age norms in the present study (see above). However, the recording frequencies in the child groups showed lower correlations $(p s<.05)$ with the recording frequencies in the adult group when compared with the .94 correlation obtained between the adult frequencies in the present study and the corresponding ones reported by Berntsen and Rubin. As expected, in this regard, the lowest correlation was found in the youngest child group.

We also directly compared the typicality scores and the idiosyncrasy scores for life scripts between the adult group and the three child groups by conducting one-way ANOVAs. Table 4 shows the means and standard deviations for these variables across groups. There was a main effect of group for both the typicality score, $F(3,226)=39.30$, partial $\eta^{2}=.343, p<.0001$, CI $=$ $385.96-417.98$, and the idiosyncrasy score, $F(3,226)=25.91$, partial $\eta^{2}=.256, p<.0001, \mathrm{CI}=1.24-1.67$.

Tamhane's post hoc comparisons showed significant differences between the four age groups, with a steady increase in typicality from third grade to fifth-sixth and eighth grade to adulthood. At the same time, the number of idiosyncratic events included in the life scripts decreased from third grade through adulthood. In third grade, $28.20 \%$ of all events were idiosyncratic events; in fifthsixth grade, $21.86 \%$ of all events were idiosyncratic; in eighth grade, $14.53 \%$ of all events were idiosyncratic; and in the adult group, only $6.56 \%$ of all life script events were idiosyncratic. Third graders mentioned, on average, significantly more idiosyncratic events in their life scripts than did eighth graders and adults, and adults mentioned significantly less idiosyncratic events than all child groups (see Table 4). Overall, the cultural life scripts of the children became more adult-like with increasing age. However, even in the oldest child group, the average typicality had not reached adult norm yet. Consistent with the analyses based on the mean scores in the child groups, significant correlations were found between age and typicality score, $r(118)=.342, p<.0001$, as well as age and idiosyncrasy score, $r(118)=-.248, p<.01$. In the adult group, no significant correlations between these variables were found, suggesting a more stable cultural life script in adulthood. 
Table 3

Frequencies (in Percentages) and Means of Estimated Ages (and Standard Deviations) for Life Script Events Recorded by Adults, Third Graders, Fifth-Sixth Graders, and Eighth Graders

\begin{tabular}{|c|c|c|c|c|c|c|c|c|}
\hline \multirow[b]{2}{*}{ Event } & \multicolumn{2}{|c|}{ Adults $(N=111)$} & \multicolumn{2}{|c|}{ Third graders $(n=42)$} & \multicolumn{2}{|c|}{$\begin{array}{l}\text { Fifth-sixth graders } \\
\qquad(n=43)\end{array}$} & \multicolumn{2}{|c|}{ Eighth graders $(n=35)$} \\
\hline & $\%$ & $M(S D)$ & $\%$ & $M(S D)$ & $\%$ & $M(S D)$ & $\%$ & $M(S D)$ \\
\hline Children & 90.10 & $29.06(2.56)$ & 40.48 & $28.41(7.67)$ & 74.42 & $27.08(4.13)$ & 77.14 & $28.34(4.08)$ \\
\hline Begin school & 87.39 & $6.05(0.36)$ & 66.67 & $6.09(0.33)$ & 72.10 & $5.95(0.50)$ & 74.29 & $5.98(0.41)$ \\
\hline Marriage & 83.79 & $28.25(4.47)$ & 45.24 & $25.50(6.47)$ & 88.37 & $25.67(3.33)$ & 68.57 & $25.82(2.69)$ \\
\hline College & 71.71 & $22.51(3.36)$ & 16.67 & $19.31(1.49)$ & 27.91 & $19.08(2.19)$ & 45.71 & $19.63(3.22)$ \\
\hline Fall in love & 53.15 & $13.71(2.33)$ & 16.67 & $12.50(3.93)$ & 30.23 & $13.23(2.31)$ & 25.71 & $12.61(1.93)$ \\
\hline Begin prekindergarten & 37.84 & $3.27(0.77)$ & 42.86 & $3.14(0.33)$ & 18.60 & $3.44(0.62)$ & 28.57 & $3.50(0.75)$ \\
\hline Confirmation & 34.23 & $13.54(0.54)$ & 16.67 & $12.86(0.69)$ & 58.14 & $13.66(0.43)$ & 54.29 & $13.71(0.38)$ \\
\hline Grandchildren & 31.53 & $57.44(5.44)$ & 0.00 & & 18.60 & $61.38(6.57)$ & 22.86 & $58.75(8.94)$ \\
\hline Retirement & 30.63 & $65.41(2.54)$ & 9.52 & $56.50(14.20)$ & 37.21 & $63.94(3.57)$ & 22.86 & $64.42(2.57)$ \\
\hline Leave home & 28.83 & $19.27(1.07)$ & 7.14 & $16.33(2.52)$ & 20.93 & $19.13(1.64)$ & 0.00 & \\
\hline Begin daycare & 25.23 & $1.77(0.99)$ & 11.90 & $1.70(0.84)$ & 0.00 & & 11.43 & $1.23(0.52)$ \\
\hline Begin senior high & 24.32 & $16.46(0.92)$ & 11.90 & $16.40(1.95)$ & 13.95 & $16.58(0.66)$ & 22.86 & $17.06(0.90)$ \\
\hline Finish senior high & 24.32 & $18.80(0.80)$ & 0.00 & & 13.95 & $19.67(1.51)$ & 8.57 & $18.50(0.87)$ \\
\hline Parents' death & 23.42 & $49.66(9.89)$ & 0.00 & & 0.00 & & 8.57 & $21.67(16.20)$ \\
\hline Baptism & 22.52 & $0.42(0.27)$ & 50.00 & $0.45(0.63)$ & 18.60 & $0.34(0.44)$ & 42.86 & $0.74(1.16)$ \\
\hline Get a job & 21.62 & $26.23(3.04)$ & 38.10 & $20.06(3.66)$ & 32.56 & $22.50(4.43)$ & 20.00 & $25.25(2.44)$ \\
\hline Siblings & 20.72 & $3.70(2.10)$ & 14.29 & $5.67(1.97)$ & 18.60 & $5.36(2.01)$ & 0.00 & \\
\hline First job & 16.22 & $25.72(3.01)$ & 0.00 & & 0.00 & & 0.00 & \\
\hline Own death & 16.22 & $84.31(8.03)$ & 16.67 & $92.33(17.99)$ & 53.49 & $86.32(10.59)$ & 62.86 & $80.42(19.52)$ \\
\hline Begin walking & 15.32 & $1.13(0.25)$ & 33.33 & $1.75(0.55)$ & 13.95 & $1.92(1.02)$ & 34.29 & $1.96(0.78)$ \\
\hline First friend & 13.51 & $6.18(3.17)$ & 14.29 & $5.25(5.33)$ & 20.93 & $6.56(1.89)$ & 17.14 & $4.50(1.94)$ \\
\hline Own birth & 12.61 & $0.00(0.00)$ & 30.95 & $0.00(0.00)$ & 23.26 & $0.00(0.00)$ & 25.71 & $0.00(0.00)$ \\
\hline Adult relationship & 12.61 & $21.07(2.20)$ & 11.90 & $25.80(13.65)$ & 16.28 & $21.21(3.11)$ & 14.29 & $24.80(3.70)$ \\
\hline Puberty & 11.71 & $13.12(1.08)$ & 0.00 & & 0.00 & & 14.29 & $13.30(1.79)$ \\
\hline Long trip & 10.81 & $19.18(1.25)$ & 0.00 & & 0.00 & & 0.00 & \\
\hline Partner's death & 9.01 & $69.50(18.17)$ & 0.00 & & 0.00 & & 0.00 & \\
\hline Career & 9.01 & $29.06(7.78)$ & 0.00 & & 0.00 & & 0.00 & \\
\hline Begin talking & 9.01 & $1.50(0.53)$ & 14.29 & $2.33(1.47)$ & 11.63 & $1.60(0.65)$ & 14.29 & $1.70(0.57)$ \\
\hline Empty nest & 8.11 & $48.50(3.66)$ & 0.00 & & 6.98 & $49.67(2.52)$ & 0.00 & \\
\hline Other's death & 7.21 & $34.13(15.04)$ & 0.00 & & 0.00 & & 0.00 & \\
\hline Own house & 7.21 & $29.69(3.58)$ & 7.14 & $25.00(8.19)$ & 9.30 & $25.67(7.50)$ & 0.00 & \\
\hline First sex & 7.21 & $16.00(1.16)$ & 0.00 & & 0.00 & & 0.00 & \\
\hline Finish ninth grade & 6.31 & $15.79(0.39)$ & 26.19 & $16.00(1.34)$ & 6.98 & $16.33(0.29)$ & 22.86 & $16.69(1.22)$ \\
\hline Divorce & 5.41 & $41.17(4.62)$ & 0.00 & & 0.00 & & 0.00 & \\
\hline Special birthdays & 5.41 & $23.33(15.11)$ & 9.52 & $33.80(41.70)$ & 0.00 & & 11.43 & $22.00(19.05)$ \\
\hline Second-last child & 5.41 & $31.00(2.10)$ & 0.00 & & 6.98 & $30.17(5.97)$ & 11.43 & $31.50(6.19)$ \\
\hline Serious disease & 4.50 & $52.40(30.37)$ & 7.14 & $42.67(38.55)$ & 0.00 & & 14.29 & $26.80(40.68)$ \\
\hline Parents' divorce & 4.50 & $5.30(2.59)$ & 0.00 & & 13.95 & $5.33(3.50)$ & 8.57 & $5.33(4.93)$ \\
\hline Become adult & 3.60 & $18.00(0.00)$ & 0.00 & & 0.00 & & 0.00 & \\
\hline Begin hobby-sport & 3.60 & $7.50(1.71)$ & 14.29 & $6.33(2.25)$ & 0.00 & & 0.00 & \\
\hline Continuation school & 3.60 & $16.00(2.71)$ & 9.52 & $16.17(0.29)$ & 0.00 & & 0.00 & \\
\hline \multicolumn{9}{|c|}{ Events mentioned by 3 or more children that were not included in the adults' life scripts } \\
\hline Driver's license & & & 23.81 & $18.63(0.92)$ & 18.60 & $18.31(0.70)$ & 11.43 & $18.00(0.00)$ \\
\hline First vacation & & & 19.05 & $6.88(3.23)$ & 20.93 & $6.61(2.83)$ & 0.00 & \\
\hline Get a pet & & & 14.29 & $21.33(15.33)$ & 6.98 & $7.00(3.60)$ & 0.00 & \\
\hline Accident & & & 7.14 & $11.33(15.37)$ & 0.00 & & 11.43 & $18.00(15.77)$ \\
\hline Learn to eat & & & 9.52 & $2.00(1.41)$ & 0.00 & & 0.00 & \\
\hline Move & & & 0.00 & & 11.63 & $7.90(3.88)$ & 0.00 & \\
\hline
\end{tabular}

Note. Percentages of "other" events mentioned less than three times in the child groups were $20.87 \%$ in the third grade, $16.70 \%$ in the fifth-sixth grades, and $12.25 \%$ in the eighth grade.

\section{Cultural Life Scripts and Their Relation to Life Narratives}

To test the hypothesis that the acquisition of a normative cultural life script plays an important role for the ability to coherently tell one's life story, but less so for the ability to coherently recount individual autobiographical events, we conducted partial correla- tions between the coherence variables and the cultural life script variables while controlling for age (see Table 5). There were significant correlations between the typicality score of cultural life scripts and the coherence measures for the life stories, but no correlation between the typicality score and the coherence score of the single-event stories. Degree of idiosyncrasy in the life script 
Table 4

Means (and Standard Deviations) for Typicality and Idiosyncrasy Scores in the Adult and Child Groups

\begin{tabular}{llc}
\hline \multicolumn{1}{c}{ Group } & $\begin{array}{c}\text { Typicality } \\
\text { score }\end{array}$ & $\begin{array}{c}\text { Idiosyncrasy } \\
\text { score }\end{array}$ \\
\hline Third graders & $272.57(126.82)$ & $2.76(2.18)$ \\
Fifth-sixth graders & $379.26(101.58)$ & $2.19(1.50)$ \\
Eighth graders & $383.22(126.33)$ & $1.53(1.63)$ \\
Adults & $466.22(77.16)$ & $0.65(0.94)$ \\
\hline
\end{tabular}

correlated negatively with coherence of single-event stories, life story endings, and number of life script events in life story, but not with the overall coherence of the life stories.

To examine whether knowledge of a cultural life script was a better predictor of life story coherence than the ability to narrate about isolated personal experiences (probed by the vacation story task), we conducted series of standard regression analyses with all life story coherence variables as dependent variables and age, life script typicality, and coherence of single-event story as predictors (see Table 6). We consistently found only age and life script typicality to be significant predictors. Only number of life script events in the life story was predicted by single-event story coherence, in addition to age and life script typicality (see Table 6).

\section{Gender Differences}

Adult group. The majority of the participants in the adult group were women, which might have lead to the establishment of a more feminine adult life script norm. However, in agreement with the results from the Erdoğan et al.'s (2008) study, we found no gender differences in the typicality scores of the adult life scripts of men and women, $t(108)=1.77$, partial $\eta^{2}=.028, p>$ .05 (CI of difference $=-59.15$ to 3.35$)$.

Child group. The proportion of girls per group increased from youngest to oldest. Thus, some of our results on age differences could be confounded by the fact that girls and boys have been found to perform differently on autobiographical memory tasks (e.g., Buckner \& Fivush, 2000; Fivush et al., 1995). We conducted a series of statistical analyses to address this potential problem. Correlational analyses controlling for gender showed virtually the same results as the ones reported earlier. We performed a multivariate ANOVA with Gender $\times$ Group (third grade, fifth-sixth grade, eighth grade) as independent variables and single-event story, life script, and life story variables as dependent variables. A main effect was found for age group on all variables $(p s<.0001$; $F$ s between 12.39 and 111.29), whereas a main effect of gender was found only for the length of single-event and life stories ( $p$ s $<.0001 ; F$ s were 15.10 and 19.76 , respectively) and global life story coherence and ending of life stories $(p \mathrm{~s}<.05 ; F$ s were 4.98 and 6.39 , respectively), as expected from previous studies of gender differences in autobiographical narratives (Buckner \& Fivush, 1998; Haden et al., 1997). An interaction was found only for length of life story, $F(2,103)=3.87$, partial $\eta^{2}=.07, p<.05$ (all other $p$ s ranged from $\geq .14$ for length of vacation story to $>.95$ for global life story coherence). Just like with the adult group, we found no effects of gender on the cultural life script variables.

\section{Discussion}

The length and coherence of children's single-event stories and life stories increased with age. We also found that the length of life stories relative to the length of single-event stories increased significantly in the older child groups. We found clear evidence in our study that third-grade children cannot produce a coherent life story, but at the same time, they were able to write coherent stories about single neutral or moderately positive autobiographical events. This finding is even more remarkable in light of some evidence that children's narratives of negative events seem to be more coherent than narratives of positive events (e.g., Ackil, van Abbema, \& Bauer, 2003; Fivush et al., 2003). A substantial minority of fifth and sixth graders were able to produce relatively coherent life stories, whereas the majority of eighth graders were able to do so. Further, there was no relationship between life story coherence and single-event story coherence when controlling for age. If general narrative coherence and life story coherence developed in parallel, then a relationship between these two types of coherence would be expected. Our study provides evidence that something more or different is needed to produce a coherent life narrative as compared with producing a coherent narrative of a single autobiographical event.

Previous studies have provided evidence that the development of general linguistic narrative coherence is important for the ability to tell a coherent story, whether it be about single autobiographical events in preschool children (Fivush et al., 1995; Hamond \& Fivush, 1991) and young adults (McAdams et al., 2006) or guided life stories (Habermas \& de Silveira, in press; Habermas \& Paha, 2001). Like the earlier studies, we found that general narrative coherence improves with age. However, at the same time, our study shows that this general narrative coherence alone cannot account for the emergence of the life story in adolescence. It is necessary, but not sufficient. On top of general narrative and linguistic competence, the present study provides evidence that the acquisition of a normative cultural life script plays an important role for the ability to coherently narrate one's life story. Establishing this evidence was possible here because, unlike previous studies, the present study measured cultural coherence separately from global life story coherence and included a baseline measure of the narrative coherence of single-event stories. The typicality of cultural life scripts increased across childhood and adolescence,

Table 5

Partial Correlations Between Coherence Variables and Cultural Life Script Normativity Variables, Controlled for Age

\begin{tabular}{lccc}
\hline \multicolumn{1}{c}{ Variable } & $\begin{array}{c}\text { Typicality } \\
\text { score }\end{array}$ & $\begin{array}{c}\text { Idiosyncrasy } \\
\text { score }\end{array}$ & $\begin{array}{c}\text { Single-event } \\
\text { story } \\
\text { coherence }\end{array}$ \\
\hline Typicality score & 1.00 & & \\
Idiosyncrasy score & $-.66^{* * *}$ & 1.00 & \\
Single-event story coherence & .04 & $-.23^{*}$ & 1.00 \\
Global life story coherence & $.28^{* *}$ & -.16 & .18 \\
Beginning life story & $.25^{* *}$ & -.16 & .06 \\
Ending life story & $.35^{* * *}$ & $-.23^{*}$ & .12 \\
Frequency of life script & $.40^{* * *}$ & $-.22^{*}$ & $.22^{*}$ \\
$\quad$ events in life stories & & & \\
\hline
\end{tabular}

${ }^{*} p<.05 . \quad{ }^{* *} p<.01 .{ }^{* * *} p<.0001$. 
Table 6

Standard Regression Analyses With Age, Life Script Typicality, and Single-Story Coherence as Predictors and Life Story Coherence Measures as Dependent Variables

\begin{tabular}{|c|c|c|c|c|c|c|c|}
\hline \multirow[b]{2}{*}{ Dependent variable } & \multicolumn{2}{|c|}{ Age } & \multicolumn{2}{|c|}{ Typicality score } & \multicolumn{2}{|c|}{$\begin{array}{l}\text { Single-event story } \\
\text { coherence }\end{array}$} & \multirow[b]{2}{*}{$R^{2}$} \\
\hline & $\beta$ & $95 \% \mathrm{CI}$ & $\beta$ & $95 \% \mathrm{CI}$ & $\beta$ & $95 \% \mathrm{CI}$ & \\
\hline Global life story coherence & $.551^{* * * *}$ & $.171, .309$ & $.214^{*}$ & $.000, .002$ & .135 & $-.021, .350$ & .523 \\
\hline Beginning life story & $.365^{* * * * *}$ & $.092, .309$ & $.237^{*}$ & $.001, .004$ & .045 & $-.220, .359$ & .267 \\
\hline Ending life story & $.430^{* * * *}$ & $.108, .257$ & $.304^{* * * *}$ & $.001, .003$ & .094 & $-.087, .311$ & .419 \\
\hline $\begin{array}{l}\text { Frequency of life script } \\
\text { events in life stories }\end{array}$ & $.474^{* * * *}$ & $.344, .675$ & $.316^{* * * *}$ & $.003, .008$ & $.175^{*}$ & $.081, .967$ & .549 \\
\hline
\end{tabular}

Note. $\quad \mathrm{CI}=$ confidence interval for $B .{ }^{*} p<.05 .{ }^{* * * *} p<.0001$.

while, at the same time, the idiosyncrasy of the life script decreased. We found that adult-like normativity of life scripts is acquired throughout childhood and well into adolescence. We also found evidence that cultural life script events and their timing seem to be acquired simultaneously; that is, children who include normative cultural life events in their life scripts also know at what point in time these events are expected to happen. These findings support the hypothesis that the cultural life script is semantic knowledge learned as a whole cognitive structure. Children seem to have established a relatively stable, but not adult-like, life script by age 12 . These findings agree with Habermas (2007), who found the largest increase in knowledge of age norms and salience of life events between ages 8 and 12. However, in our sample, 14-yearolds had life scripts deviating significantly from the adult norm, partly because this age group included more than twice as many idiosyncratic events in their life scripts than did adults.

There were significant correlations between the typicality of the cultural life scripts and life story coherence, but no correlation was found between the coherence of single-event stories and the typicality of the cultural life script. This correlational pattern was maintained in analyses controlling for the age of the participants. Thus, children with less typical and more idiosyncratic life scripts generally wrote less coherent life stories.

This suggests that the acquisition of a cultural life script may be an important precondition for the ability to generate a personal life story. As we have argued in the introduction (see also Habermas \& Bluck, 2000), with the acquisition of a cultural life script, the child learns that a personal life span is not an open temporal space but a time span that is prestructured in terms of certain culturally expected events marking the transition from one life time period and social role to another. Acquiring a cultural life script is to acquire a generic life story structure on the basis of which the child's own personal life story can be generated. The child learns to evaluate his or her personal life experiences against a normative cultural past and to plan his or her future with regard to a culturally expected future. In the present study, none of the third graders, only about $24 \%$ of fifth and sixth graders, and almost $60 \%$ of the eighth graders finished their life stories with an evaluation of the past or an outlook into the future (cf. Table 2) and adult-like typicality of the life script was positively correlated with the degree of evaluation and future outlook in the life story. Following these findings, it seems likely that the acquisition of a cultural life script may also be closely connected to the ability to mentally project oneself into the personal future and envision potential personal events (Suddendorf \& Corballis, 1997). The development of such future mental time travel in childhood and its underlying mechanisms are important questions for future research. In a broader theoretical context, the present findings on the interconnectedness of cultural life scripts and personal life story abilities agree with the emphasis on cultural learning as a unique vehicle for human cognitive development (Herrmann, Call, HernàndezLloreda, Hare, \& Tomasello, 2007; Tomasello, 1997; Tomasello et al., 1993) as well as the view that autobiographical memory relies on social cultural factors (Nelson \& Fivush, 2004).

\section{The Effects of Personal Experience}

Because our study is cross-sectional, we cannot completely rule out that the direction of causality might be the reverse of the one we assume. Under this alternative interpretation, children develop the ability to tell their life stories, which in turn enables them to generate a life script. If so, then we should expect children to use their own lives as templates for producing cultural life scripts. We explored this question by counting the number of events in an individual life story that directly mapped onto events mentioned by the same person in the life script task. The overlap of events between individually matching life stories and life scripts was much smaller $(M=1.19, S D=1.20)$ than the number of life script events in life stories overlapping with the adult generic life script $(M=3.64, S D=2.12), t(108)=15.17, p<.0001$ (CI for the difference $=-2.77$ to -2.13 ). Thus, children often included events from the adult life script in their life story that they had not themselves mentioned as part of the life script task. These results seem to indicate that children do not simply use their personal life as a template for a cultural life script but rather acquire a cultural life script through interactions with their social and cultural surroundings, which in turn helps to structure the life story. Further support for this conclusion can be drawn from comparing the events mentioned in the life stories with the cultural life script events generated by the children. In both the fifth-sixth-grade group and the eighth-grade group, parents' divorce and the death of a grandparent were among the most frequently mentioned events in the life stories. However, the death of a grandparent was not part of children's life scripts (see Table 3), and even though "parents' divorce" was part of the children's life script, only 1 of the 20 children who wrote about their parents' divorce in the life 
story also mentioned this event as part of the life script. Thus, almost all of the children in our study who had actually experienced the divorce of their parents did not see it as part of the cultural life script. The latter is consistent with the idea that life scripts are acquired through cultural transmission rather than developed from personal experience and that they mainly contain positive events expected to happen to the majority of people (cf. Berntsen \& Rubin, 2004).

\section{Motivation}

From a more speculative perspective, motivational factors are likely to contribute as well. One of the reasons for the increase of coherence in life stories by early and middle adolescence might be that the interest in writing one's life story increased considerably from about 11-12 years onward. Not only were the older children's life stories longer than their single-event stories, but it was also quite noticeable during data collection that the older children were much more eager to write their life story and many of them considered the vacation story task as just another class assignment. In contrast, the younger participants found the life story task quite daunting but enjoyed narrating an event from their fall vacations. The adolescents' genuine interest to tell their life stories could also be detected in their concerns with confidentiality. They wanted to be sure before starting the life story task that only the experimenter, but neither their parents nor their teacher, would get to read their life stories. Consequently, many adolescents wrote about difficult phases in their lives. This observation is in agreement with the idea presented in the introduction to the study that anonymously written life stories are more likely to consist of more personal and truthful autobiographical memories than life stories elicited by an interviewer.

\section{Gender}

As could be expected from the literature, we found that girls' narratives were longer and more coherent than boys' narratives (Buckner \& Fivush, 1998), whereas no gender differences were found in regard to the cultural life script. Neither children nor adults differed in their life scripts as a function of their gender. This agrees with the only study so far that has looked at gender differences concerning life scripts (Erdoğan et al., 2008). Further, the absence of gender differences in cultural life scripts agrees with research on the distribution of autobiographical memories across the life span. Rubin, Schulkind, and Rahhal (1999) found no gender differences on the distribution of autobiographical memories across the life span in an American sample. This was true for word-cued memories as well as for the five most important memories elicited from participants. If cultural life scripts indeed structure recall from autobiographical memory, as more studies suggest (e.g., Berntsen \& Rubin, 2004; Collins et al., 2007; Rubin \& Berntsen, 2003), then the absence of gender differences in cultural life scripts would be expected to result in the absence of gender differences on the distribution of autobiographical memories across the life span, which is in accordance with Rubin et al.'s (1999) findings.

\section{Written Versus Oral}

We have argued that eliciting written life stories is the preferable method when studying life stories in children and adolescents.
However, could it be that third graders prompted to orally tell their life story instead of trying to write it would have been better able to do so? This is unlikely for various reasons. First, in a pilot study, we tried to elicit life stories orally from third graders. The results were, despite probing by the interviewer, much the same as in the present study. Second, the third graders had no difficulty writing about a single autobiographical event, and third, the instructions for both narrative tasks were designed specifically with the youngest participants in mind.

In summary, the present study found evidence for the claim made by Habermas and Bluck (2000) that the ability to compose a coherent life story only emerges in adolescence. By directly comparing narratives of single autobiographical events and life stories, we were able to provide evidence that some aspects of global life story coherence develop separately from narrative coherence. Our findings suggest that some of these extra-narrative abilities that are needed to tell a life story depend on the acquisition of a cultural life script.

\section{References}

Ackil, J. K., van Abbema, D. L., \& Bauer, P. J. (2003). After the storm: Enduring differences in mother-child recollections of traumatic and nontraumatic events. Journal of Experimental Child Psychology, 84, 286-309.

Berntsen, D., \& Rubin, D. C. (2002). Emotionally charged autobiographical memories across the life span: The recall of happy, sad, traumatic, and involuntary memories. Psychology and Aging, 17, 636-652.

Berntsen, D., \& Rubin, D. C. (2004). Cultural life scripts structure recall from autobiographical memory. Memory \& Cognition, 32, 427-442.

Buckner, J. P., \& Fivush, R. (1998). Gender and self in children's autobiographical narratives. Applied Cognitive Psychology, 12, 407-429.

Buckner, J. P., \& Fivush, R. (2000). Gendered themes in family reminiscing. Memory, 8, 401-412.

Collins, K. A., Pillemer, D. B., Ivcevic, Z., \& Gooze, R. A. (2007). Cultural scripts guide recall of intensely positive life events. Memory \& Cognition, 35, 651-659.

de Vries, B., Birren, J. E., \& Deutchman, D. E. (1990). Adult development through guided autobiography: The family context. Family Relations, 39, 3-7.

Eaton, K. (2005, April). Young adults' narratives for positive and negative experiences across different reporting conditions: An integrative analysis. Poster presented at the biannual meeting of the Society for Research in Child Development, Atlanta, GA.

Erdoğan, A., Baran, B., Avlar, B., Cağlar Taş, A., \& Tekcan, A. I. (2008). On the persistence of positive events in life scripts. Applied Cognitive Psychology, 22, 95-112.

Erikson, E. H. (1968). Identity, youth, and crisis. New York: Norton.

Fivush, R., Haden, C., \& Adam, S. (1995). Structure and coherence of preschoolers' personal narratives over time: Implications for childhood amnesia. Journal of Experimental Cognitive Psychology, 60, 32-50.

Fivush, R., Hazzard, A., McDermott Sales, J., Sarfati, D., \& Brown, T. (2003). Creating coherence out of chaos? Children's narratives of emotionally positive and negative events. Applied Cognitive Psychology, 17, $1-19$.

Friedman, W. J. (1992). Children's time memory: The development of a differentiated past. Cognitive Development, 7, 171-187.

Friedman, W. J., \& Lyon, T. D. (2005). Development of temporalreconstructive abilities. Child Development, 76, 1202-1216.

Habermas, T. (2006). "Kann ich auch ganz, ganz am Anfang anfangen, als ich noch ganz klein war?" Wie Kinder und Jugendliche lernen, erzählte Lebensgeschichten zu eröffnen und beenden ["Can I start at the very beginning, when I was really little?" How children and adolescents learn 
how to open and end life stories]. In H. Welzer \& H. J. Markowitsch (Eds.), Warum Menschen sich erinnern: Fortschritte der interdisziplinären Gedächtnisforschung (pp. 256-275). Stuttgart, Germany: KlettCotta.

Habermas, T. (2007). How to tell a life: The development of the cultural concept of biography. Journal of Cognition and Development, 8, 1-31.

Habermas, T., \& Bluck, S. (2000). Getting a life: The emergence of the life story in adolescence. Psychological Bulletin, 126, 748-769.

Habermas, T., \& de Silveira, C. (in press). The development of global coherence in life narratives across adolescence: Temporal, causal, and thematic aspects. Developmental Psychology.

Habermas, T., \& Paha, C. (2001). The development of coherence in adolescents' life narratives. Narrative Inquiry, 11, 35-54.

Haden, C. A., Haine, R. A., \& Fivush, R. (1997). Developing narrative structure in parent-child reminiscing across the preschool years. Developmental Psychology, 33, 295-307.

Hamond, N. R., \& Fivush, R. (1991). Memories of Mickey Mouse: Young children recount their trip to Disneyworld. Cognitive Development, 6, 433-448.

Herrmann, E., Call, J., Hernàndez-Lloreda, M. V., Hare, B., \& Tomasello, M. (2007, September 7). Humans have evolved specialized skills of social cognition: The cultural intelligence hypothesis. Science, 371(5843), 1360-1366.

Hudson, J. A., \& Fivush, R. (1991). As time goes by: Sixth graders remember a kindergarten experience. Applied Cognitive Psychology, 5, 347-360.

Luborsky, M. R. (1993). The romance with personal meaning in gerontology: Cultural aspects of life themes. Gerontologist, 33, 445-452.

McAdams, D. P. (1996). Personality, modernity, and the storied self: A contemporary framework for studying persons. Psychological Inquiry, 7, 295-321.

McAdams, D. P. (2001). The psychology of life stories. Review of General Psychology, 5, 100-122.

McAdams, D. P. (2006). The problem of narrative coherence. Journal of Constructivist Psychology, 19, 109-125.

McAdams, D. P., Bauer, J. J., Sakaeda, A. R., Anyidoho, N. A., Machado, M. A., Magrino-Failla, K., et al. (2006). Continuity and change in the life story: A longitudinal study of autobiographical memories in emerging adulthood. Journal of Personality, 74, 1371-1400.

McCormack, T., \& Hoerl, C. (1999). Memory and temporal perspective: The role of temporal frameworks in memory development. Developmental Review, 19, 154-182.

McLean, K. C. (2005). Late adolescent identity development: Narrative meaning making and memory telling. Developmental Psychology, 41, 683-691.

Nelson, K. (1988). The ontogeny of memory for real events. In U. Neisser \& E. Winograd (Eds.), Remembering reconsidered: Ecological and traditional approaches to the study of memory (pp. 244-276). New York: Cambridge University Press.

Nelson, K. (2003). Self and social functions: Individual autobiographical memory and collective narrative. Memory, 11, 125-136.

Nelson, K., \& Fivush, R. (2004). The emergence of autobiographical memory: A social cultural developmental theory. Psychological Review, $111,486-511$.

Neugarten, B. L., Moore, J. W., \& Lowe, J. C. (1965). Age norms, age constraints, and adult socialization. The American Journal of Sociology, 70, 710-717.

Peterson, C. (2002). Children's long-term memory for autobiographical events. Developmental Review, 22, 370-402.

Peterson, C., \& McCabe, A. (1983). Developmental psycholinguistics: Three ways of looking at a child's narrative. New York: Plenum.

Pillemer, D. B., Picariello, M. L., \& Pruett, J. C. (1994). Very long term memories of a salient preschool event. Applied Cognitive Psychology, 8, 95-106.

Reese, E. (2002). Social factors in the development of autobiographical memory: The state of the art. Social Development, 11, 124-142.

Rubin, D. C., \& Berntsen, D. (2003). Life scripts help to maintain autobiographical memories of highly positive, but not highly negative, events. Memory \& Cognition, 31, 1-14.

Rubin, D. C., Berntsen, D., \& Hutson, M. (2008). The normative and the personal life: Individual differences in life scripts and life stories among Danish and U.S.A. undergraduates. Manuscript submitted for publication.

Rubin, D. C., Schulkind, M. D., \& Rahhal, T. A. (1999). A study of gender differences in autobiographical memory: Broken down by age and sex. Journal of Adult Development, 6, 61-71.

Schank, R. C., \& Abelson, R. P. (1977). Scripts, plans, and knowledge. In P. N. Johnson-Laird \& P. C. Wason (Eds.), Thinking: Readings in cognitive science (pp. 421-435). Cambridge, England: Cambridge University Press.

Singer, J. A. (2004). Narrative identity and meaning making across the adult lifespan: An introduction. Journal of Personality 72, 437-460.

Singer, J. A., \& Bluck, S. (2001). New perspectives on autobiographical memory: The integration of narrative processing and autobiographical reasoning. Review of General Psychology, 5, 91-99.

Smith, R., Heuerman, M., Wilson, B. M., \& Proctor, A. (2003). Analysis of normal discourse patterns. Brain and Cognition, 53, 368-371.

Suddendorf, T., \& Corballis, M. C. (1997). Mental time travel and the evolution of the human mind. Genetic, Social and General Psychology Monographs, 123, 133-167.

Tomasello, M. (1997). Culture and cognitive development. Current Directions in Psychological Science, 9, 37-40.

Tomasello, M., Kruger, A., \& Ratner, H. (1993). Cultural learning. Behavioral Brain Sciences, 16, 495-552.

van Abbema, D. L., \& Bauer, P. J. (2005). Autobiographical memory in middle childhood: Recollections of the recent and distant past. Memory, $13,829-845$.

Wilson, B. M., Smith, R., \& Proctor, A. (2001). The validity of cognitive distance in oral and written discourse. Brain and Cognition, 46, 304 307.

Received May 29, 2007

Revision received February 21, 2008 Accepted March 16, 2008 